\title{
DIVERSIDAD SEXUAL, SALUD Y CIUDADANÍA
}

\author{
Carlos F. Cáceres ${ }^{1, a}$, Víctor A. Talavera ${ }^{1, b}$, Rafael Mazín Reynoso ${ }^{2, c}$
}

\begin{abstract}
RESUMEN
Las orientaciones sexuales no heterosexuales dejaron de ser consideradas como patológicas por la Asociación Psiquiátrica Americana en 1973 y por la Organización Mundial de la Salud en 1990. Sin embargo, en opinión de los autores, en América Latina estos cambios no se han traducido ni en una atención en salud inclusiva ni en el reconocimiento como ciudadanos plenos de las personas no heterosexuales. Este documento tiene como propósito brindar un panorama teórico referente a sexualidad, proponiendo condiciones apropiadas que permitan encarar exitosamente los desafíos que se presentan. Así, se expone el papel regulador que ejerce la medicina sobre la sexualidad, hecho que ha generado reacciones tanto académicas como políticas. Se señala el progresivo cambio dado en las ciencias médicas: desde considerar patológica la sexualidad no heterosexual hasta ofrecerle, al menos en teoría, inclusión. Se describen los impactos negativos que un ambiente social adverso genera en un individuo. Finalmente, se proponen acciones concretas a tomar para brindar mejores servicios de salud a las y los ciudadanos no heterosexuales.
\end{abstract}

Palabras clave: Sexualidad; Homosexualidad; Diversidad cultural; Derechos humanos; Políticas públicas (fuente: DeCS BIREME).

\section{SEXUAL DIVERSITY, HEALTH AND CITIZENSHIP}

\begin{abstract}
Non-heterosexual sexual orientations are no longer considered pathological by the American Psychiatric Association since 1973 and the World Health Organization since 1990. However, to many authors, these changes in Latin America have not been translated into an inclusive healthcare provision or the recognition of non-heterosexual citizens with full rights. The purpose of this document is to provide a theoretical view regarding sexuality, proposing proper conditions that allow to successfully facing arising challenges. Thus, the regulating role of medicine over sexuality has been exposed, which has led to both academic and political reactions. The progressive change given in medical science has been pointed out: from considering non-heterosexual sexuality to be pathological to offering, in theory at least, inclusion. The negative impacts of what an adverse social environment causes to an individual are described. Finally, specific actions are proposed to provide better health services to non-heterosexual citizens.
\end{abstract}

Key words: Sexuality; Homosexuality; Cultural diversity; Human rights; Public policies (source: MeSH NLM).

\section{INTRODUCCIÓN}

Durante la última década, en muchos países del mundo se ha visto la necesidad de prestar mayor atención a la salud de las comunidades no heterosexuales ${ }^{(1,2)}$. Esto ha resultado, en parte, en el hecho de que en este período se hayan dado pasos claros en el reconocimiento y respeto de personas y comunidades definidas por su sexualidad no heterosexual (o su no conformidad con el género asignado a su sexo biológico), cuya expresión más importante ha sido, tal vez, la aprobación del matrimonio igualitario en un creciente número de naciones, como Holanda, Bélgica, España, Portugal, Francia, Canadá, varios estados de Estados Unidos, Nueva Zelanda, Argentina, Uruguay, e incluso Ciudad de México. Además, hay formas no igualitarias de matrimonio, unión civil o reconocimiento de derechos conyugales vigentes en un número adicional de países (3). Asimismo, en países que partieron de legislaciones mucho más restrictivas, el mayor cambio se ha dado en la descriminalización de las relaciones homosexuales, como ocurrió en India en $2009{ }^{(4)}$.

\footnotetext{
Unidad de Salud, Sexualidad y Desarrollo Humano, Universidad Peruana Cayetano Heredia. Lima, Perú.

Programa Regional de VIH, Organización Panamericana de la Salud. Washington DC, USA.

a Médico, magister y doctor en Salud Pública, especialista en Salud Internacional; ${ }^{\mathrm{b}}$ estudiante de Psicología; ${ }^{\mathrm{c}}$ médico, magister en Salud Pública, y especialista en Educación Sexual.

Recibido: 08-07-2013 Aprobado: 02-10-13

Citar como: Cáceres CF, Talavera VA, Mazín Reynoso R. Diversidad sexual, salud y ciudadanía. Rev Peru Med Exp Salud Publica. 2013;30(4):698-704.
} 
En los lugares donde ha ocurrido, la aprobación del matrimonio igualitario, así como la viabilización del cambio de nombre y género ante la ley, han significado, de facto, la plena incorporación a la sociedad de los ciudadanos con orientaciones no heterosexuales e identidades de género alternativas, al menos en el plano formal. Una consecuencia de ello ha sido que la ausencia histórica de servicios de salud orientados a atender las necesidades de estas poblaciones se haga cada vez más evidente ${ }^{(5)}$. Esta ausencia se explica, sin duda, porque las orientaciones no heterosexuales eran consideradas patológicas hasta 1973 (según la Asociación Psiquiátrica Americana) (6) y hasta 1990 (según la Clasificación Internacional de Enfermedades de la Organización Mundial de la Salud [OMS], oficializada en 1992) ${ }^{(7)}$. Existe, además, un creciente consenso sobre el retiro de la llamada disforia de género ${ }^{(8)}$, como se alude a la identidad transgénero, de la nueva edición de dicha clasificación, anunciada para $2015^{\left.{ }^{9}\right)}$. A pesar de los cambios señalados, un número significativo de profesionales de la salud continúa viendo a estas poblaciones como enfermas e incluso prefieren evitar tratarlas, particularmente cuando el estigma de esta forma de sexualidad se superpone con el estigma asociado al VIH. El número de instrumentos legales internacionales que comprometen a los estados, con la meta de responder a estos retos, es cada vez mayor. También existen, por parte de la OMS y de la Organización Panamericana de la Salud, para nuestra región, un número creciente de recomendaciones técnicas y herramientas ${ }^{(10,11)}$ para atender algunas de estas necesidades en un plazo breve.

El Perú se cuenta entre los países de América Latina que menos ha avanzado en el reconocimiento legal de estas comunidades, y que solo responde a su salud en lo que concierne al VIH/SIDA, sin estar preparado para atender su salud integral con una perspectiva de ciudadanía plena. En el presente texto se abordan estos conceptos y se presentan los mencionados instrumentos internacionales, señalando dónde están disponibles, y se sugiere la necesidad de incorporar otros. Confiamos en que las nuevas generaciones de profesionales, que se han formado en contextos sociales más globalizados, irán teniendo cada vez mayor disposición para responder a necesidades impostergables para con este sector de ciudadanos secularmente excluido.

\section{SALUD, SEXUALIDAD Y CIENCIA}

\section{EL CONCEPTO DE SALUD}

Salud es un estado de completo bienestar físico, mental y social, no solo la ausencia de enfermedad (12). Por lo tanto, el gozar de buena salud no es solamente no tener enfermedad, incapacidad o disfunción; es también disfrutar de un bienestar razonable en todas las facetas de nuestra vida personal e interpersonal. Garantizar el derecho a la salud significa proteger el derecho inalienable a la vida del que gozan todos los seres humanos.

El derecho a la salud va más allá de garantizar prevención y curación de enfermedades: debe asegurar estándares de vida que garanticen el bienestar en todas sus dimensiones ${ }^{(5)}$.

\section{ORIENTACIÓN SEXUAL, IDENTIDAD DE GÉNERO E IDENTIDAD SEXUAL, INTERSEXUALIDAD}

La orientación sexual se refiere a si una persona siente atracción sexual por personas del otro género, de su mismo género, o de ambos géneros ${ }^{(13)}$. Kinsey desarrolló una escala que solicitaba a los sujetos clasificar su orientación sexual entre 0 (solo personas del mismo sexo) y 6 (solo personas del otro sexo), donde 3 equivalía a "igualmente atraído por personas de ambos sexos", y las otras cifras, a distintos grados de bisexualidad con predominio de una orientación.

La identidad de género se refiere a la identificación de una persona como hombre o mujer, independientemente de su sexo biológico ${ }^{(13)}$. Actualmente, si una persona nacida como hombre se identifica como mujer, puede esperar que se le llame mujer trans (en el caso contrario hablamos de hombre trans).

La identidad sexual se refiere a la identificación de una persona (según identidad de género, como hombre o mujer; y según su orientación sexual, hacia personas del otro, del mismo o de ambos géneros), como hombre heterosexual, gay o bisexual; o como mujer heterosexual, lesbiana o bisexual ${ }^{(14)}$. Nótese que esta clasificación es independiente de la identidad de género, de modo que una mujer trans atraída por hombres sería una mujer trans heterosexual; igualmente, un hombre trans atraído por hombres sería un hombre trans gay; y así sucesivamente, clasificación que puede parecer contraintuitiva, pero que resulta de una aplicación lógica de las categorías integradas.

La intersexualidad se refiere a situaciones en las que, por alteraciones genéticas $u$ hormonales, un infante puede nacer con caracteres sexuales primarios que no corresponden a su sexo genético, o puede ser genéticamente hermafrodita, con características físicas que lo reflejan ${ }^{(15)}$. Esta situación incluye una gama de condiciones en las que los genitales son ambiguos o en los que existe hermafroditismo, las que tradicionalmente 
la medicina trató de 'definir' a temprana edad, mediante cirugía. En los últimos años, el movimiento de personas intersexuales ha reclamado que la elección del género debe dejarse a las mismas personas cuando estas puedan decidir en su adolescencia, práctica que actualmente se plantea al interior de la disciplina médica ${ }^{(16)}$.

\section{UNA RELECTURA DEL DISCURSO CIENTÍFICO SOBRE SEXUALIDAD EN LOS SIGLOS XIX YXX}

Durante la primera mitad del siglo XIX, la naciente medicina moderna se preocupó por regular mejor el campo de la sexualidad normal, lo que algunos interpretan como una necesidad planteada por la revolución industrial ${ }^{(17,18)}$. Esta sexualidad normal fue definida como una práctica heterosexual conyugal enfocada en la reproducción (y definida por la penetración vaginal casi exclusivamente). Otras prácticas heterosexuales, así como las prácticas homosexuales y autoeróticas, fueron etiquetadas como psicopatías sexuales ${ }^{(19)}$ y se plantearon tratamientos para ellas, dado que no eran vistas como prácticas elegidas sino como expresiones de alguna enfermedad mental.

Esta visión prevaleció hasta fines del siglo XIX, cuando Sigmund Freud impuso su teoría de la libido, según la cual una sexualidad multipotencial es vista como una fuerza natural (perversidad polimorfa) que la educación controla, aunque con costos como las neurosis (incluida la histeria y las compulsiones) ${ }^{(20)}$. Ainicios del siglo XX un grupo de médicos y sexólogos (como Ellis y Hirschfeld) comenzaron a proponer un enfoque benigno de la homosexualidad, y los primeros antropólogos (Boas, Malinowski, Benedict, Mead) proporcionaron evidencia de que las diferencias transculturales desestabilizaban lo que se consideraba normalidad sexual, permitiendo una relativización de esta concepción ${ }^{(18)}$. Hacia mediados del siglo XX, en Estados Unidos, los estudios de Kinsey también plantean a la sociedad de postguerra que ciertas prácticas sexuales (incluida la homosexualidad) eran mucho más comunes que lo que normalmente se reconocía ${ }^{(21)}$.

Durante varias décadas, pensadores freudianos marxistas como Reich, Fromm y Marcuse reinterpretan lo que Freud consideró perversidad polimorfa, planteando que su control educativo es una práctica de opresión sexual (que debía enfrentarse a través de una "revolución sexual"). Dicha línea de pensamiento, que inspiró las revueltas de fines de los años sesenta, es una de las bases del surgimiento de los movimientos feminista y gay, y de sus contrapartes académicas. Una revuelta en el bar transexual de Stonewall en Nueva York un 28 de junio de 1969, contra los abusos policiales, marca el inicio de un movimiento gay no dispuesto a aceptar concesiones, y que se ha ido globalizando en los últimos 30 años ${ }^{(22,23)}$. Actualmente, este movimiento reconoce la diversidad en su interior, y se autodenomina LGBTI (lésbico-gay-bisexual-transgénero-intersex).

\section{ASPECTOS REGULATORIOS Y SITUACIÓN DEL CONOCIMIENTO}

Aunque en 1952 la Asociación Psiquiátrica Americana (APA) incluyó en su primer "Manual diagnóstico y estadístico de los trastornos mentales" (DSM) a la homosexualidad como un trastorno, en 1973 esta fue retirada del DSM con una declaración: La homosexualidad per se no conlleva impedimento en juicio, estabilidad, confiabilidad o capacidades sociales y vocacionales ${ }^{(6)}$. En 1975, la Asociación Psicológica Americana adoptó una posición similar a la APA y urgió a todos los profesionales de la salud a: Asumir un rol protagónico en la remoción del estigma que durante largo tiempo ha asociado las orientaciones homosexuales con la enfermedad mental.

La OMS en el Manual de Clasificación Internacional de Enfermedades (CIE o ICD-9, 1977), todavía incluía a la homosexualidad como una enfermedad mental. Sin embargo, el 17 de mayo de 1990, urgida por la XLIII Asamblea Mundial de la Salud, retiró la homosexualidad de la CIE-10, razón por la cual el 17 de mayo se ha convertido en una fecha emblemática en la que se celebra el Día Mundial contra la Homofobia, Lesbofobia y Transfobia. Aunque la no conformidad de género aún está incluida en la ICD como un trastorno, existe una considerable probabilidad que sea desclasificada en la CIE-11, a publicarse en 2015, con base en revisiones sistemáticas de evidencia que se han desarrollado recientemente, que todavía no han sido publicadas. En otros países del mundo estos procesos también se han ido dando, aunque algo más lentamente. Por ejemplo, el "Manual de clasificación de trastornos mentales" de la República Popular de China retiró la homosexualidad de sus páginas en 2001

En la actualidad se considera que las atracciones, orientaciones y comportamientos sexuales entre personas del mismo sexo son variantes normales del comportamiento sexual humano. Asimismo, la atracción y prácticas sexuales entre personas del mismo sexo pueden ocurrir en el contexto de una variedad de orientaciones e identidades (24). Las personas homosexuales pueden tener vidas muy satisfactorias; no existen estudios empíricos, ni literatura revisada por pares que apoye las teorías que asocian la orientación homosexual o identidad transgenérica con disfunción familiar o trauma infantil ${ }^{(25)}$. Es la estigmatización, y no la orientación no heterosexual en sí, lo que genera tensión 
y estrés a lo largo de la vida y consecuente impacto sobre la salud mental. Sin duda, hoy en día las poblaciones LGBTI enfrentan factores múltiples que afectan su salud y bienestar en América Latina y globalmente.

De otro lado, los tratamientos de reconversión de la orientación sexual no tienen ningún fundamento científico y sí una gran base de prejuicio y discriminación causantes de grave daño emocional (26). Actualmente, son considerados procedimientos no éticos por la OMS, pues parten de la presunción de una enfermedad por tratarse, no son efectivos en cambiar la orientación sexual, y suelen generar sufrimiento en las personas que se someten a ellas. Así, en el año 2009, un grupo de especialistas de la APA publicó una revisión sistemática de toda la literatura científica existente en inglés desde el año 1960 hasta el año 2007, donde se concluye que las personas homosexuales y bisexuales forman parejas y familias saludables y que el estigma es la principal causa de malestar en estas personas ${ }^{(25)}$. Cabe resaltar el progresivo declive, en países desarrollados, de las terapias reparativas. Asimismo, se deben mencionar las disculpas públicas ofrecidas por Robert Spitzer ${ }^{(27)}$, quien publicó un controversial estudio, en el que afirmaba que era posible dejar de ser homosexual y convertirse en heterosexual, a pesar de que en 1973 fue uno de los promotores de la despatologización de la homosexualidad. Alan Chambers, presidente de Exodus International, también se disculpó recientemente por el "devastador daño hecho a otras personas”, aceptando él mismo que, aunque ha estado por largo tiempo casado con una mujer, no había podido suprimir su orientación homosexual, y que esto no puede imponérsele a otras personas, porque causa "daño inconmensurable" (28).

\section{LA PSICONEUROINMUNOLOGÍA: EXPLICANDO EL IMPACTO DE LOS PROBLEMAS SOCIALES SOBRE LA SALUD}

Gracias a investigaciones desarrolladas en las últimas décadas, el estrés de la exclusión social se puede comprender mejor que antes. Las agresiones del entorno producen una respuesta hormonal que lleva a estrés, ansiedad y depresión; fenómenos que desencadenan señales neurológicas que, en algunos casos, deprimen la respuesta inmunológica de la persona y la hacen susceptible a una serie de procesos patológicos, incluyendo infecciones, alteraciones funcionales, y neoplasias ${ }^{(29)}$. Otra consecuencia de estos problemas puede darse en la esfera psicológica; por ejemplo, una mayor incidencia de uso problemático de alcohol y drogas, o de intentos de suicidio o suicidio efectivo.

Considerando las agresiones directas $\mathrm{O}$ indirectas que las personas de las comunidades LGBTI pueden percibir hacia sí o su forma de vivir, a lo largo de su vida la existencia de problemas reactivos psicosociales es bastante frecuente, y estos pueden, por lo señalado, asociarse a una mayor incidencia de otros problemas. Las agresiones y manifestaciones de hostilidad más frecuentes incluyen situaciones descritas como de desdén, desafecto, desapego, maltrato, inequidad, discriminación, tratamiento degradante e indigno, persecución, tortura, y negación de derechos. Adicionalmente, puede haber una exposición a agentes patógenos especiales, en relación con las características de las exposiciones. Por ejemplo, la exclusión sexual puede llevar a prácticas sexuales clandestinas y riesgosas, con mayor exposición a infecciones de trasmisión sexual (ITS), VIH/SIDA y hepatitis viral, y con menor búsqueda de medidas preventivas y de atención médica. En estos casos, el ambiente es poco favorable para la prevención, con un control limitado sobre los riesgos (lo que se relaciona con el concepto de vulnerabilidad).

Hace algunos años se acuñó el concepto de sindemia para referirse a epidemias paralelas ${ }^{(29,30)}$ y se considera que estas son frecuentes en poblaciones LGBT. Por ejemplo, la exclusión social y el menor acceso a servicios múltiples lleva a los hombres gay a epidemias de depresión, uso problemático de alcohol y drogas, ITS y VIH/SIDA.

\section{DIVERSIDAD SEXUAL, EXCLUSIÓN SOCIAL Y POLIITICAS PÚBLICAS}

\section{CONSECUENCIAS DE LA PATOLOGIZACIÓN DE ALGUNAS SEXUALIDADES}

La patologización de algunas sexualidades puede tener claras consecuencias sobre las opciones de vida privada y pública (laboral, profesional) y experiencia de salud de las personas. Por ejemplo, puede llevar a dictámenes civiles, penales, laborales, y administrativos desventajosos; puede exponer a las personas a prácticas médicas nocivas; suele conllevar una limitación de los derechos ciudadanos de las personas (incluidos los relativos a custodia, adopción, convivencia); y suele ser una justificación para la opresión, el escarnio social y el maltrato. Todo esto es incompatible con un estado de bienestar completo en todas las facetas de la vida personal e interpersonal de las personas LGBTI (31).

\section{LA INSUFICIENTE RESPUESTA DEL SECTOR SALUD}

Como se dijo, una gran limitación de esta respuesta está en la ausencia de servicios de salud pensados en responder a necesidades específicas de esta comunidad, o en la limitada voluntad o capacidad para ofrecerle servicios 
Tabla 1. Condiciones para una respuesta efectiva a las necesidades específicas de las comunidades LGBTI

\begin{abstract}
Condiciones
Reconocer a las comunidades y colectivos LGBTI. de protección y cuidado de la salud. $y$ afectadas.

Establecer una política de no tolerancia a la intolerancia. salud. poblaciones. y agencias internacionales.

Fomentar el intercambio de experiencias y promover una cultura de respeto.

adecuados. Todo esto llega a tener una expresión negativa extrema en situaciones en las que hay demora sustancial en el otorgamiento de servicios, humillaciones públicas, y abuso verbal y físico. Puede haber también abuso sexual, procedimientos no consentidos, e incluso tortura. Las condiciones necesarias para una respuesta efectiva que debieran ser consideradas por los profesionales se detallan en la Tabla 1.
\end{abstract}

Promover el desarrollo de liderazgos en su interior, y favorecer la participación de organizaciones de la sociedad civil en acciones

Conformar grupos y coaliciones de apoyo, denunciando las violaciones de derechos, y protegiendo a las personas más vulnerables

Desarrollar lineamientos claros para definir estrategias de atención de los colectivos LGBTI. Son necesarias sinergias interprogramáticas, con acciones sincrónicas, convergencia de agendas y actividades, y uso adecuado de la evidencia.

Mejorar la recolección y análisis de información estratégica relevante, y sensibilizar y capacitar a los miembros del equipo de

Desarrollar o adaptar protocolos que faciliten la atención de miembros de estas comunidades en los servicios de salud, como las que han planteado la OMS ${ }^{(32)}$ y los Blueprints de la OPS para $\mathrm{HSH}^{\left({ }^{(3)}\right)}$ y para población trans ${ }^{(34)}$, en lo concerniente a la atención de VIH e ITS principalmente; no obstante, se reconoce la ausencia de lineamientos que aborden la salud integral de estas

Sensibilizar a legisladores, formuladores de políticas y tomadores de decisiones, a través de acciones de abogacía desde redes

LGBTI: lesbianas, gays, bisexuales, transgénero e intersex, OMS: Organización Mundial de la Salud, OPS: Organización Panamericana de la Salud HSH: hombres que tienen sexo con hombres, VIH: virus de inmunodeficiencia humana, ITS: infecciones de transmisión sexual

\section{CONCLUSIONES Y DESAFÍOS INMEDIATOS}

Pese a la desclasificación de la orientación no heterosexual como enfermedad por parte de la OMS en 1990, los sistemas de salud no reconocen, en la práctica, la legítima existencia de las comunidades, no solo al no responder plenamente a sus problemas específicos de salud, sino inclusive al no ofrecerles atención adecuada en muchos servicios generales. Muchos profesionales, además, continúan considerando a sus miembros como personas afectadas por una enfermedad psicológica.

La evidencia muestra, sin embargo, que los problemas de salud mental de personas LGBTI (que incluyen la depresión, el uso de sustancias y el suicidio, entre los más comunes), se explican fundamentalmente por la exclusión social y la exposición sistemática a discriminación, violencia y agresión por parte del entorno.
Los desafíos en el futuro inmediato nos plantean la necesidad de actuar sobre las múltiples expresiones de exclusión social y ciudadanía restringida de las comunidades LGBTI, incluyendo: terminar con la discriminación, maltrato y violencia en la sociedad, la familia y el entorno cercano, mediante cambios legales adecuados, y asegurando el cumplimiento de la ley y la sensibilización de los operadores de justicia; luchar contra la indiferencia y discriminación persistentes en el sector salud, mediante el desarrollo de servicios adecuados para atender las necesidades generales y específicas de estas comunidades; y terminar con la falta de oportunidades educativas, laborales y de participación social como generadora de inequidades, marginalidad y pobreza, donde corresponde, claramente, una acción multisectorial para identificar y resolver las barreras a la plena incorporación ciudadana de los miembros de estas comunidades, secularmente ignoradas y postergadas.

Contribuciones de autoría: CC concibió, diseñó y redactó el artículo con base en información clave aportada por RM, quien realizó además varios aportes en la concepción de la idea original. VT colaboró en la interpretación y redacción parcial del texto, y de la revisión crítica. Todos los autores aprobaron la versión final.

Fuentes de financiamiento: autofinanciado.

Conflictos de interés: los autores declaran no tener conflictos de interés. 


\section{REFERENCIAS BIBLIOGRÁFICAS}

1. Open Society Institute; International Centre for Human Rights Education La salud sexual y los derechos humanos: una guía de recursos [Internet]. Nueva York:OSI/ Equitas; 2007 [citado el 12 de julio de 2013]. Disponible en: http://www. equalpartners.info/PDFDocuments/ SpaPartialGuideSexualHealth.pdf

2. Organización Panamericana de la Salud (OPS). Salud Sexual para el Milenio. Declaración y documento técnico. Washington, DC: OPS; 2009.

3. Agence France-Presse. Los 14 países donde el matrimonio homosexual es legal. ABC.es [Internet]. 23 de abril de 2013 [citado el 12 de julio de 2013]; sociedad. Disponible en: http:// www.abc.es/sociedad/20130423/ abci-paises-matrimonio-homosexualaprobado-201304231837.html

4. Elmundo.es. India legaliza la homosexualidad [Internet]. Agence France-Presse, 3 de julio de 2009 [citado el 12 de julio de 2013]. Disponible en: http://www. elmundo.es/elmundo/2009/07/02/ solidaridad/1246513907.htm

5. Oficina de las Naciones Unidas Alto Comisionado para los Derechos Humanos; Organización Mundial de la Salud. El derecho a la salud. Folleto informativo No 31. Geneva: OMS; 2008.

6. American Psychiatric Association. Position Statement on Homosexuality and Civil Rights. Am J Psychiat. 1973;131(4):497.

7. World Health Organization. ICD-10. Classification of mental and behavioral disorders: clinical descriptions and diagnostic guidelines. Geneva: World Health Organization; 1992.

8. World Professional Association for Transgender Health (WPATH). Executive Summary: WPATH consensus process regarding transgender and transsexual-related diagnoses in ICD-11 [Internet]. Minneapolis: WPATH: 31 de mayo de 2013 [citado el 15 de Julio de 2013]. Disponible en: http://www.wpath.org/ documents/WPATH\%20ICD-11\%20 Consensus\%20Report_Executive\%20 Summary_7-7-13.pdf

9. World Health Organization. The International Classification of
Diseases 11th Revision is due by 2015. [Internet]. Geneva: WHO; 2013 [citado el 08 de Julio de 2013]. Disponible en: http://www.who.int/ classifications/icd/revision/en/

10. Organización Panamericana de la Salud. La OPS/OMS advierte que el estigma y la discriminación afectan la salud de lesbianas, gays, bisexuales y trans. PAHO press releases [Internet]. Washington, DC: OPS; 16 de mayo de 2013 [citado el 05 de julio de 2013]. Disponible en: http://new.paho. org/hq/index.php?option $=$ com_co ntent \&view $=$ article $\&$ id $=8670$ $\% 3$ Astigma-and-discriminationjeopardize-the-health-of-lesbiansgays-bisexuals-and-transgenderpeople\&catid $=740 \% 3$ Anews-pressreleases\&Itemid $=1926 \&$ lang $=$ es

11. Organización Panamericana de la Salud. "Curas" para una enfermedad que no existe. PAHO press releases [Internet]. Washington, DC: OPS; 17 de mayo de 2012 [citado el 05 de julio de 2013]. Disponible en: http://www.paho.org/ arg/index.php?option=com_conten t\&view $=$ article\&id $=960 \&$ catid $=711$ :- - - a c ceso-universal-vih / sida\&Itemid $=286$

12. World Health Organization. WHO definition of health [Internet]. Geneva: WHO; 2003 [citado el 4 de julio de 2013]. Disponible en: http://www. who.int/about/definition/en/print. html

13. American Psychological Association (APA). Definition of terms: Sex, Gender, Gender Identity, Sexual Orientation [Internet]. Washington, DC: APA; 2011 [citado el 4 de julio de 2013]. Disponible en: http://www. apa.org/pi/lgbt/resources/sexualitydefinitions.pdf

14. Cass VC. Homosexual identity formation: a theoretical model. J Homosex. 1979;4(3):219-35.

15. Intersex Society of North America (ISNA). What is intersex? [Internet]. Rohnert Park, CA: ISNA; 2008 [citado el 4 de Julio de 2013]. Disponible en: http://www.isna.org/faq/what_is_ intersex

16. Chase Ch. What is the agenda of the intersex patient advocacy movement? In: First World Congress: Hormonal and Genetic Basis of Sexual
Differentiation Disorders; 17-18 de mayo de 2002. Arizona, Estados Unidos, 2002.

17. Foucault M. Historia de la sexualidad I: La voluntad de saber. $3^{a}$ ed. México: Siglo XXI; 2011.

18. Weeks J. Sexuality and its discontents. Meanings, myths \& modern sexualities. New York: Routledge; 1985.

19. Krafft-Ebing R. Psychopathia Sexualis. Philadelphia: The F.A. Davis Company; 1894.

20. Strachey J, editor. Standard Edition of the Complete Psychological Works of Sigmund Freud. London: Hogarth Press; 1986

21. Kinsey AC, Pomery WB, Martin CE. Sexual behavior in the human male. Philadelphia: W.B. Saunders; 1948.

22. Carter D. Stonewall: The riots that sparked the Gay Revolution. New York: St. Martin's Press; 2004.

23. Chan PhC. Protection of sexual minorities since stonewall: progress and stalemate in developed and developing countries. Abingdon: Routledge; 2012.

24. Cáceres CF, Rosasco AM. The margin has many sides: diversity among gay and homosexually active men in Lima. Cult Health Sex. 1999;1(3):261-75.

25. APA Task Force on Appropriate Therapeutic Responses to Sexual Orientation. Report of the Task Force on Appropriate Therapeutic Responses to Sexual Orientation. Washington D.C: American Psychological Association; 2009.

26. Haldeman DC. The pseudo-science of sexual orientation conversion therapy. ANGLES. 1999;4(1):1-4.

27. Carey B. Psychiatry giant Sorry for Backing Gay "Cure". The New York Times [Internet]. 19 de mayo de 2012 [citado el 4 de julio de 2013]. Disponible en: http://www. nytimes.com/2012/05/19/health/ dr-robert-1-spitzer-noted-psychiatristapologizes-for-study-on-gay-cure. html? pagewanted $=$ all\&_r $r=0$

28. Chambers A. I Am Sorry. Exodus International [Internet]. 19 de junio de 2013 [citado el 10 de julio de 2013]. Disponible en: http://wespeaklove.org/ wp-content/uploads/2013/08/I_Am_ Sorry_Exodus_International.png 
29. Aral SO, Fenton KA, Lipshuz JA, editores. The new public health and STD/HIV prevention: personal, public and health systems approaches. New York: Springer Sciences+Business Media; 2013.

30. Stall R, Mills TC, Williamson J, Hart T, Greenwood G, Paul J, et al. Association of co-occurring psychosocial health problems and increased vulnerability to HIV/AIDS among urban men who have sex with men. Am J Public Health. 2003;93(6):939-42.

31. American Psychological Association. APA policy statements on lesbian, gay, bisexual \& transgender concerns
[Internet]. Washington, DC: APA; 2011 [citado el 05 de julio de 2013]. Disponible en: http://www.apa.org/ about/policy/booklet.pdf

32. Organización Mundial de la Salud. Prevención y tratamiento de la infección por el VIH y otras infecciones de transmisión sexual entre hombres que tienen sexo con hombres y personas transgénero: recomendaciones para un enfoque de salud pública. Washington, DC: OPS; 2012.

33. Organización Panamericana de la Salud. Proyecto para la provisión de cuidado integral a los hombres gay y otros hombres que tienen sexo con hombres (HSH) en América Latina y el Caribe. Washintgton, DC: OPS; 2010.

34. Organización Panamericana de la Salud. Por la salud de las personas trans: elementos para el desarrollo de la atención integral de personas trans y sus comunidades en Latinoamérica y el Caribe. En prensa.

\section{Correspondencia: Carlos F. Cáceres}

Dirección: Av. Armendáriz 445, Lima 18, Perú.

Teléfono: (51) 2033300

Correoelectrónico: carlos.caceres@upch.pe

\section{AHORA EN EL INS}

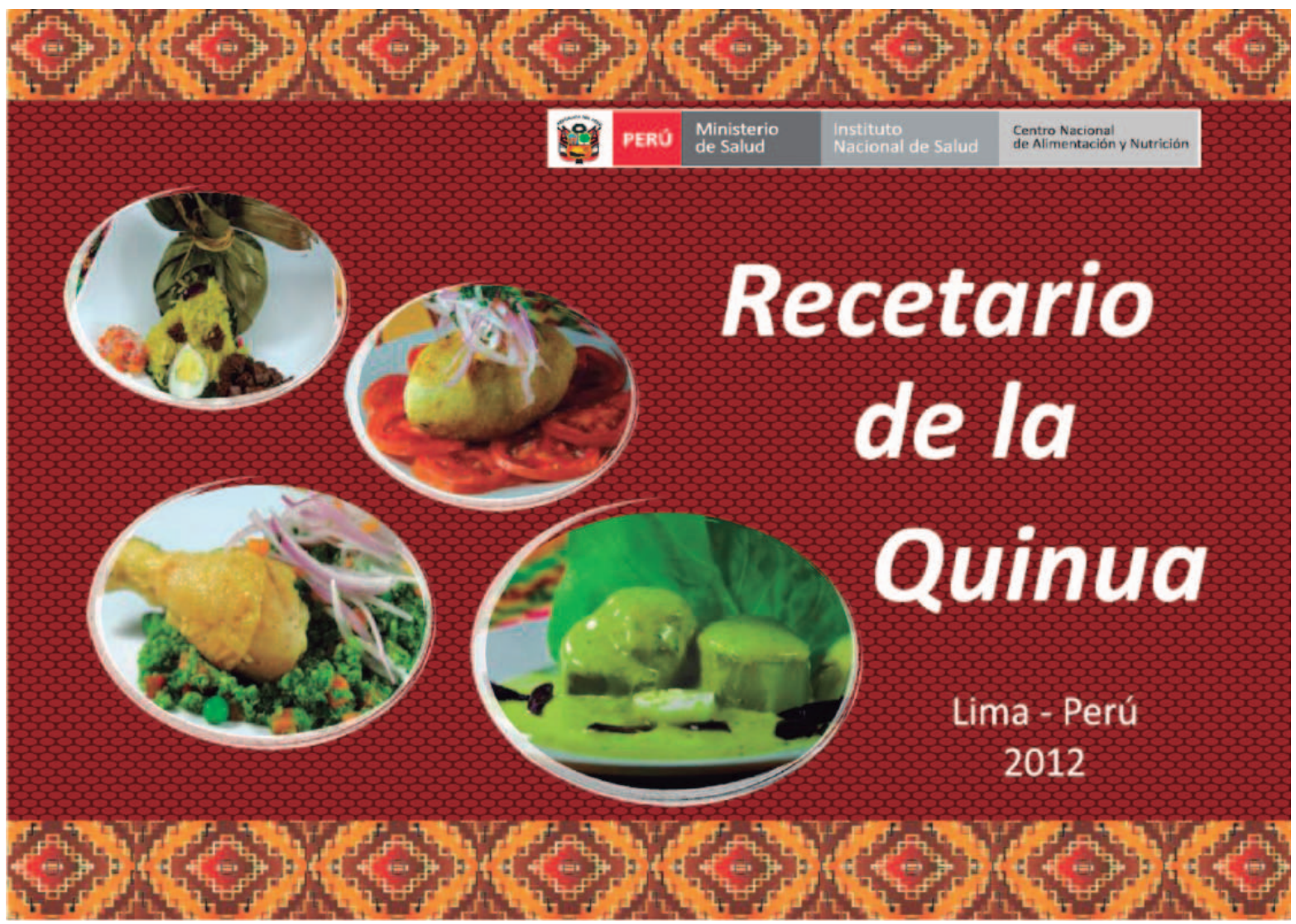

Disponible en:

http://www.ins.gob.pe/repositorioaps/0/0/zop/zona_publicacion_01/RECETARIO\%20DE\%20QUINUA\%20INSp.pdf 